\title{
Trimester-Specific Reference Ranges for Saturated, Monounsaturated and Polyunsaturated Fatty Acids in Serum of Pregnant Women: A Cohort Study from the ECLIPSES Group
}

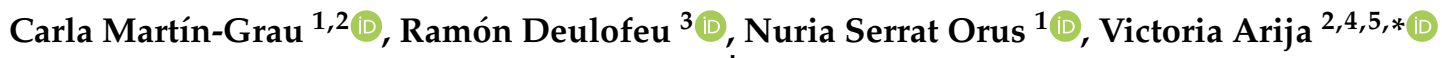 \\ and on behalf of the ECLIPSES Study Group ${ }^{\dagger}$ \\ 1 Clinical Chemistry and Laboratory Medicine, Institut Català de la Salut, Generalitat de Catalunya, \\ University Hospital Joan XXIII, 43005 Tarragona, Spain; carla.mg324@gmail.com (C.M.-G.); \\ nserrat.tarte.ics@gencat.cat (N.S.O.) \\ 2 Nutrition and Mental Health Research Group (NUTRISAM), Faculty of Medicine and Health Science, \\ Universitat Rovira i Virgili, 43201 Reus, Spain \\ 3 Department of Biochemistry and Molecular Genetics, Biomedical Diagnostic Center, Hospital Clínic \\ Universitari, CiberEHM, 08036 Barcelona, Spain; deulofeu@clinic.cat \\ 4 Pere Virgili Institute for Health Research (IISPV), Universitat Rovira i Virgili, 43003 Tarragona, Spain \\ 5 Tarragona-Reus Research Support Unit, Jordi Gol Primary Care Research Institute, 43003 Tarragona, Spain \\ * Correspondence: victoria.arija@urv.cat; Tel.: +34-977-759-334 \\ $+\quad$ The ECLIPSES Study Group is provided in the Acknowledgments.
}

Citation: Martín-Grau, C.; Deulofeu, R.; Serrat Orus, N.; Arija, V.; on behalf of the ECLIPSES Study Group. Trimester-Specific Reference Ranges for Saturated,

Monounsaturated and

Polyunsaturated Fatty Acids in Serum of Pregnant Women: A Cohort Study from the ECLIPSES Group. Nutrients 2021, 13, 4037. https:// doi.org/10.3390/nu13114037

Academic Editor: Martine Armand

Received: 15 October 2021

Accepted: 10 November 2021

Published: 12 November 2021

Publisher's Note: MDPI stays neutral with regard to jurisdictional claims in published maps and institutional affiliations.

Copyright: (c) 2021 by the authors. Licensee MDPI, Basel, Switzerland. This article is an open access article distributed under the terms and conditions of the Creative Commons Attribution (CC BY) license (https:/ / creativecommons.org/licenses/by/ $4.0 /)$.

\begin{abstract}
In the course of pregnancy, increasing importance is being placed on maintaining optimal fatty acid (FA) levels and particularly n-3 PUFAs to ensure correct fetal development. However, reference ranges for FA have been reported in only a few studies. Our objective is to provide quantitative reference intervals for SFAs, MUFAs, and PUFAs (n-6 and n-3) in a large population of healthy pregnant women from a developed country. A prospective study of pregnant women ( $n=479)$ was conducted from the first trimester (T1) to the third trimester (T3). A total of 11 fatty acids were analyzed in serum by gas chromatography mass spectrometry and were expressed as absolute $(\mu \mathrm{mol} / \mathrm{L})$ and relative (percentage of total FA) concentration units. Serum concentrations of SFAs, MUFAs, n-6 PUFAs, n-3 PUFAs, various FA ratios, and the EFA index were determined. The reference intervals (2.5/97.5 percentiles) in absolute values from T1 ranged from 1884.32 to $8802.81 \mu \mathrm{mol} / \mathrm{L}$ for SFAs, from 959.91 to $2979.46 \mu \mathrm{mol} / \mathrm{L}$ for MUFAs, from 2325.77 to $7735.74 \mu \mathrm{mol} / \mathrm{L}$ for n-6 PUFAs, and from 129.01 to $495.58 \mu \mathrm{mol} / \mathrm{L}$ for n-3 PUFAs. These intervals mainly include the values of other studies from European populations. However, reference ranges vary according to some maternal factors. The FA levels proposed, obtained from a large sample of pregnant women, will be a useful tool for assessing the degree of adequacy of FAs in pregnant women and will help to carry out dietary interventions based on certain maternal factors.
\end{abstract}

Keywords: individual c14-c24; fatty acid status; maternal nutrition; pregnancy; reference intervals

\section{Introduction}

Maternal diet during the periconceptional, pregnancy and lactation period is important for both mother and child [1,2], and specifically, the fatty acid (FA) levels play a crucial role during pregnancy [3-5]. Eicosapentaenoic acid (EPA) and docosahexaenoic acid (DHA) are specially critical regarding central nervous system, retinal photoreceptors, and immune systems development [6]. Correct concentrations (or status) both in the first trimester (T1) and the third trimester (T3) of pregnancy are of great importance considering their role during the onset of neurogenesis $[7,8]$, and the fetal brain development [8]. Consequently, maternal serum FA levels change in the course of pregnancy, depending on fetal requirements. In this context, it is necessary to monitor FA concentration values throughout pregnancy so that deficiency can be detected. Based on this, reference ranges are the most 
useful tool to evaluate the adequacy of nutritional status [9], and even in future studies these values can be used to identify women who are at risk of an adverse health outcome.

In the little literature that does exist, reference ranges for individualized FA in serum are normally reported in non-pregnant adults [10-13]. Apparently, North European populations have a higher content of total saturated fatty acids (SFAs) and a lower content of monounsaturated fatty acids (MUFAs) circulating in serum [13-15] than North American populations [11,12]. Furthermore, in populations of healthy pregnant women, serum reference values have only been described in a Brazilian cohort of 225 pregnant women [16] and in a Norwegian cohort of 247 pregnant women [17]. All these populations show different dietary patterns from the one in our study. In the Mediterranean population, the composition of the diet is well-known for its low content of SFAs, and high content of MUFAs and n-3 PUFAs, mainly derived from olive oil and fish consumption [18,19]. Consequently, reference values of circulating FA should be measured in different populations based on their lifestyle.

Additionally, FAs can be measured in serum, erythrocyte, or adipose tissue. It is well-recognized that FA levels in serum reflect short-term intake [20], and are more representative of the current dietary habits of subjects [21]. However, other authors have analyzed FAs in the erythrocyte membrane for assessing FA status as it reflects the last 30-60 days of intake [7,17] so comparisons among studies are difficult. In previous studies, the analysis of an individual FA may be found expressed either quantitatively or qualitatively as a relative percentage of total FAs. Without standardizing the criteria for providing an appropriate reference range, there is a great risk of misinterpretation. In this regard, the problem of presenting findings such as relative values is that they are difficult to compare since percent composition values depend on the set of individual FAs investigated [10,11]. By contrast, absolute values are not dependent upon the relative abundance of other FAs and the measurement of an individual FA reduces analysis costs thus could be included in routine clinical practice of laboratories and not be limited to research studies. A quantitative approach may potentially be more appropriate for analyzing whether FAs are associated with the risk of an adverse health outcome.

To support the measurement of individual FAs, the use of $n-6 / n-3$, arachidonic acid (AA)/EPA, AA/(EPA + DHA) and linoleic acid (LA)/Dihomo- $\gamma$-linolenic acid (DHGLA) ratios and the essential fatty acid (EFA) index are also helpful in assessing the degree of adequacy of FAs. The increase in the AA/EPA ratio has been positively correlated with proinflammatory eicosanoids and associated with metabolic diseases [22,23]. An imbalance between AA and EPA + DHA was found to be associated to preterm delivery $[22,24]$. High LA/DHGLA ratios indicate an enzymatic deficit of delta-6-desaturase activity. Delta-6desaturase is predominantly involved in the PUFA biosynthesis pathway and converts LA and alpha-lipoic acid (ALA) into their metabolites DHGLA, AA, EPA and DHA [3,7], although it can also catalyze palmitic acid and stearic acid thus affecting MUFA levels [25]. The $n-6 / n-3$ ratio reflects the predominant PUFA family in serum and the formation of inflammatory mediators, which play important roles in pregnancy pathologies $[26,27]$. According to the available data, no study has reported reference intervals for serum n-6/n-3, $\mathrm{AA} / \mathrm{EPA}, \mathrm{AA} /(\mathrm{EPA}+\mathrm{DHA})$ and LA/DHGLA ratios, or the EFA index in pregnant women. While providing these reference ranges, future studies will allow correlating newborn adverse outcomes with an individual FA abnormality or any ratio imbalance.

To our knowledge, no study has evaluated maternal FAs status and ratios in European Mediterranean countries, and including a large majority of SFAs, MUFAs and PUFAs. Considering the lack of reference intervals for pregnant women in the literature and the absence of interpretative criteria, the aim of this study was to provide the reference intervals for serum SFA, MUFA and PUFA (n- 6 and n-3) in a large population of healthy pregnant women from a Mediterranean country in T1 and T3 of gestation, expressed both as concentrations and as percentages of the total concentration of FA in serum samples. 


\section{Materials and Methods}

\subsection{Population and Study Design}

A prospective study of pregnant women was conducted from T1 to T3 (12 \pm 0.5 and $36 \pm 0.4$ gestational week, respectively). Participants were healthy pregnant women from the ECLIPSES study $[28,29]$ which is a randomized, triple-blind clinical trial of different doses of iron supplementation $(20 \mathrm{mg} /$ day, $40 \mathrm{mg} /$ day $80 \mathrm{mg} /$ day), registered in ClinicalTrials.gov (accessed on 9 November 2021) identification number NCT03196882, and in the European Union (EU) Clinical Trial Register, EUCTR-2012-005480-28. The present study was approved by the Clinical Research Ethics Committee of the Jordi Gol Institute for Primary Care Research (IDIAP) and the Pere Virgili Institute for Health Research (IISPV). Informed consent was obtained from all individuals included in this study.

The flow of participants and data is outlined in Figure 1. The inclusion criteria were as follows: healthy adult women older than 18 years at $\leq 12$ weeks of gestation, who were able to understand the local languages (Spanish or Catalan) and the characteristics of the study. Some of the medium risk criteria and all the high risk criteria described in the Catalan pregnancy monitoring protocol [30] were used as exclusion criteria such as multiple pregnancies, underweight, morbid obesity, previous severe disease (immunosuppression), or any chronic disease that could affect nutritional development (cancer, pre-gestational diabetes mellitus, malabsorption, chronic hepatitis and liver cirrhosis).

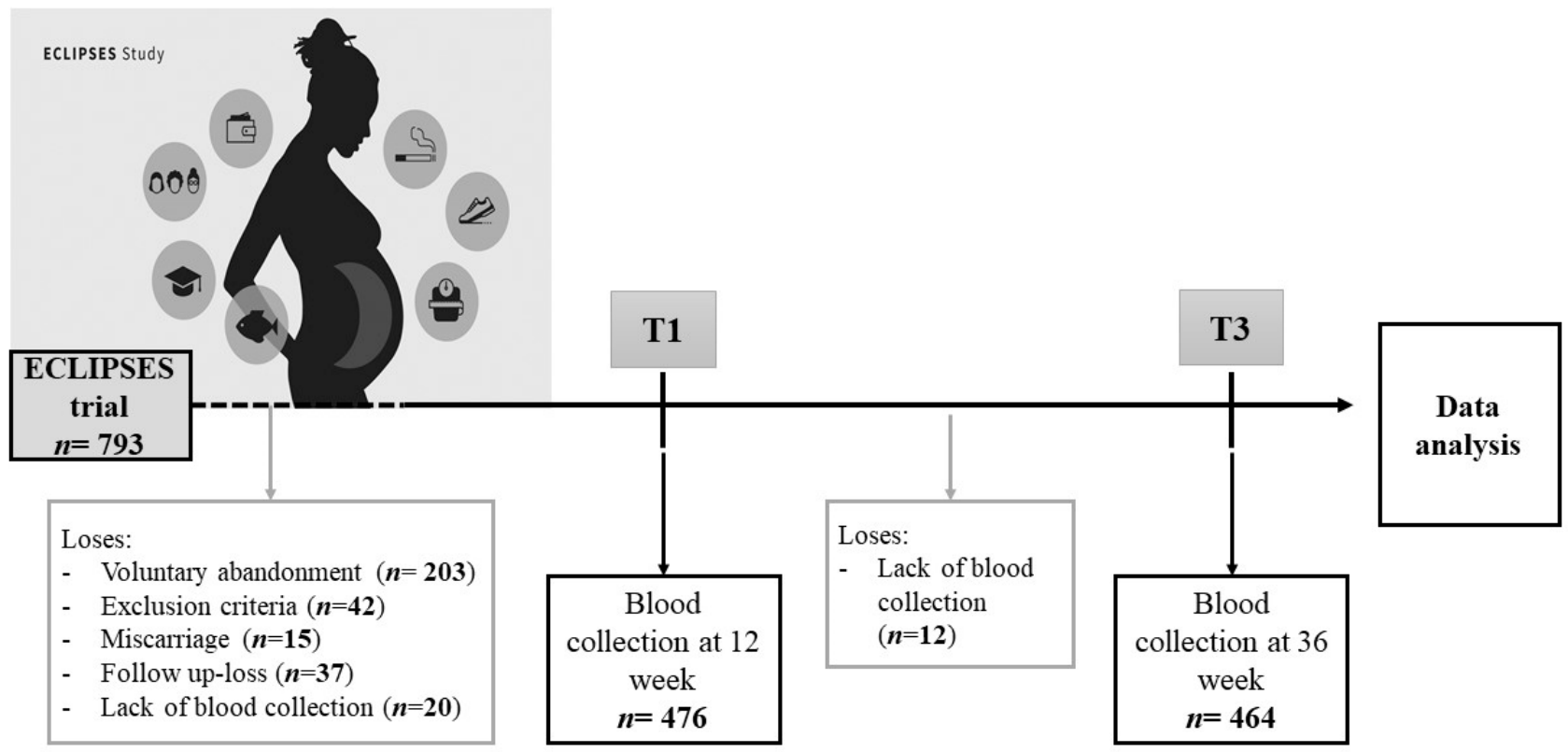

Figure 1. Flow chart of the study population.

The medical, socioeconomic, educational level and lifestyle information is shown in Table 1. The socioeconomic level was calculated by using the Catalan classification of occupations (CCO-2011) [31] such as student, employed and unemployed. The education level was classified as primary (low), high school (medium) and university studies or more (high). Physical activity was assessed by using the International Physical Activity Questionnaire [32] and was summed to obtain the total physical activity in metabolic equivalents (METs)/week. Based on total physical activity, participants were classified into 3 levels: low ( $<600$ METs /week), moderate ( $\geq 600-2999$ METs/week) and high ( $\geq 3000 \mathrm{METs} /$ week). BMI was calculated and classified by following WHO criteria [33]: normal weight $\left(B M I=18.5-24.9 \mathrm{~kg} / \mathrm{m}^{2}\right)$, overweight $\left(B M I=25.0-29.9 \mathrm{~kg} / \mathrm{m}^{2}\right)$ and obesity $\left(\mathrm{BMI} \geq 30 \mathrm{~kg} / \mathrm{m}^{2}\right)$ at T1. The Spanish diet quality index (SQDI) [34] was estimated from nine food groups (protein foods, dairy foods, cereals, fruits, vegetables, oil, legumes, tubers, sweets). A score was obtained ranging from 0 points (low quality diet) to 18 points (high 
quality diet). Women were then classified into two categories: low-medium diet quality (score from 0 to 10) and high diet quality (score from 11 to 18). Further information can be found in our previous paper [29].

Table 1. Sociodemographic and lifestyle characteristics of pregnant women $(n=455)$.

\begin{tabular}{|c|c|}
\hline General Characteristics & Summary Statistics \\
\hline Maternal age (years) $^{a}$ & $30.6 \pm 5.01$ \\
\hline Country of origin, Spain (\%) & 84.1 \\
\hline Primipara (\%) & 37.5 \\
\hline Gestational age (weeks) $^{a}$ & $39.8 \pm 1.08$ \\
\hline \multicolumn{2}{|l|}{ BMI $\left(\mathrm{kg} / \mathrm{m}^{2}\right)$ at first trimester $(\%)$} \\
\hline 18.5-24.9 (normal weight) & $62.2(22.10 \pm 1.76)^{\mathrm{a}}$ \\
\hline 25.0-29.9 (overweight) & $25.3(27.32 \pm 1.33)^{a}$ \\
\hline$\geq 30$ (obesity) & $12.5(33.31 \pm 2.91)^{\mathrm{a}}$ \\
\hline Gestational weight gain $(\mathrm{kg})^{a}$ & $10.86 \pm 3.66$ \\
\hline \multicolumn{2}{|l|}{ Maternal educational level (\%) } \\
\hline Low (primary or less) & 30.1 \\
\hline Medium (high school) & 38.3 \\
\hline High (university or more) & 31.6 \\
\hline \multicolumn{2}{|l|}{ Occupation (\%) } \\
\hline Student & 2.4 \\
\hline Employed & 87.1 \\
\hline Unemployed & 10.5 \\
\hline \multicolumn{2}{|l|}{ Smoking status (\%) } \\
\hline Smoker & 15.3 \\
\hline Non-Smoker & 69.5 \\
\hline Ex-Smoker & 15.3 \\
\hline Maternal alcohol consumption (\%) & 14 \\
\hline \multicolumn{2}{|l|}{ Physical Activity (METs/week) (\%) } \\
\hline Low $(<600)$ & 56.4 \\
\hline Moderate $(\geq 600-2999)$ & 39.4 \\
\hline $\operatorname{High}(\geq 3000)$ & 4.2 \\
\hline SQDI (score) ${ }^{a}$ & $9.73 \pm 2.64$ \\
\hline
\end{tabular}

\subsection{Sample Preparation and GC-MS Conditions}

\subsubsection{Extraction, Transfer and Storage of Biological Samples}

Serum samples were collected both $\mathrm{T} 1$ and $\mathrm{T} 3$ after fasting into $7.5 \mathrm{~mL}$ tubes without anticoagulant and were left without mixing for $30 \mathrm{~min}$ at room temperature to enable coagulation. The serum was separated by centrifugation and stored into aliquots of $500 \mu \mathrm{L}$ at $-80{ }^{\circ} \mathrm{C}$ inside the BioBank. Samples were thawed at the end of the study and processed simultaneously to minimize inter-batch variation [28].

\subsubsection{Sample Preparation and GC-MS Conditions}

The method starts with a $50 \mu \mathrm{L}$ serum sample mixed with IS and the derivatization reagent (chloroform and methanolic hydrochloric acid). Then the sample is heated and mixed at $80{ }^{\circ} \mathrm{C}$ for $2 \mathrm{~h}$. In this step, the lipidic fraction in blood or serum consisting of free FAs, sterol esters, glycerol esters (mainly triglycerides), and phospholipids were hydrolyzed and methylated into fatty acid methyl esters (FAMEs). Three sequential heating and mixing steps are done every 10 min during a 30-min period. After this, a $100 \mu \mathrm{L}$ of 
iso-octane was added and the fatty acid methyl esters were extracted by a liquid-liquid extraction using hexane, and analyzed by gas chromatography mass spectrometry (GC-MS) combination using the 7890A GC coupled to triple quadrupole MS QQQ 7000 Series ${ }^{\circledR}$ (Agilent Technologies Inc., Santa Clara, CA, USA). Chromatographic analysis was based on David et al. [35] to determine the 36 FAMEs included in the Food Industry FAME Mix (Restek Corporation). Briefly, the FAMEs were separated into a high-polarity column $(100 \mathrm{~m} \times 250 \mu \mathrm{m} \times 0.25 \mu \mathrm{m})(\mathrm{HP}-88$ column, Agilent Technologies) using a temperature program ranging between 140 and $240{ }^{\circ} \mathrm{C}$ at a $10^{\circ} / \mathrm{min}$ pace using helium as the carrier gas at $1 \mathrm{~mL} / \mathrm{min}$. Ionization was carried out by electronic impact $(70 \mathrm{eV})$, and the mass analyzer was operated in Selected Ion Monitoring (SIM) mode. The CG-MS system was controlled by the Agilent MassHunter ${ }^{\circledR}$ Workstation.

\subsection{Data Analysis}

A total of 36 FAs were analyzed although FA values below the limit of detection were not shown. In addition, a selection of FAs is presented such as the sum of the total saturated ( $\Sigma$ total SFA $=$ C:12 + C:14 + C:16 + C18:0), total monounsaturated ( total MUFA $=\mathrm{C} 16: 1 \mathrm{n}-7+\mathrm{C} 18: 1 \mathrm{n}-9)$, total n-6 polyunsaturated $(\Sigma$ total n-6 PUFA $=\mathrm{C} 18: 2 \mathrm{n}-6+\mathrm{C} 20: 3 \mathrm{n}-6$ + C20:4n-6) and total n-3 polyunsaturated ( $\sum$ total n-3 PUFA $=$ C20:5n-3 + C22:6n-3) fatty acids; furthermore, some index were also calculated such as the n- 6 to n-3 fatty acids ratio ( $\Sigma$ total n-6 PUFA/ $\Sigma$ total n-3 PUFA) and the essential fatty acids (EFA) index (sum of the (essential) n-3 and n-6 PUFAs/sum of the (non-essential) n-7 and n-9 FAs), which reflects the overall EFA status.

FAs were expressed as absolute ( $\mu \mathrm{mol} / \mathrm{L}$ ) or relative (percentage of total FA) concentration units. The method for calculating the percentage of total fatty acids is based on total FA concentration. To assist in the evaluation of overall nutritional status, reference intervals were determined following the Clinical and Laboratory Standards Institute (CLSI) C28- $\mathrm{A} 3$ guidelines [9] and represented the central $95 \%$ of the tested population (being the $2.5 \%$ and $97.5 \%$ confidence intervals the lower and upper limits, respectively). The FA values were analyzed using Agilent MassHunter ${ }^{\circledR}$ Quantitative Analysis B.06 (Agilent Technologies Inc., Santa Clara, CA, USA). The results were expressed as mean \pm standard deviation (SD) for normally distributed data. In this study, z-score analysis was used to detect outlier values in the population data [36]. An absolute z-score value above or below \pm 3.29 is considered to be an outlier when the sample size is $>100$ [37]. Correlations between the absolute and relative concentrations of FA in serum were computed using Pearson's correlation coefficient test. All multiple linear regression models were performed using the ENTER method for total FAs, total SFA, total MUFA, total n-6 PUFA, total n-3 PUFA, LA, DHA and AA to evaluate the relation between maternal factors and FA levels. The models were adjusted for maternal factors, such as maternal age, occupation (student, employed, unemployed), educational level (low, medium, high), ethnicity (Spanish, non-Spanish women), parity (no, yes), initial BMI $\left(\mathrm{Kg} / \mathrm{m}^{2}\right)$, gestational weight gain $\left(\mathrm{Kg} / \mathrm{m}^{2}\right)$, maternal smoking status (no, yes), maternal alcohol consumption (no, yes), physical activity in METs/week (score), and SQDI (score). Data was processed using the statistical software package SPSS version 25.0 for Windows and Microsoft Excel 2016 (Microsoft Corporation, Redmond, WA, USA). A $p$-value $<0.05$ was considered to be statistically significant.

\section{Results}

\subsection{Participants' Characteristics}

The general characteristics of the pregnant women participating in the ECLIPSES study are given in Table 1. A total of 455/476 women answered the questions about sociodemographic and lifestyle characteristics. Most women had a medium educational level $(38.3 \%)$ and were employed $(87.1 \%)$. The maternal age was $30.6 \pm 5.01$ years old. The participants reported that $15.3 \%$ smoked and $14 \%$ drank at the beginning of pregnancy. Regarding physical activity, $56.4 \%$ of the women had a low level. 


\subsection{Fatty Acid Status in Serum of Pregnant Women}

This study determined saturated, mono- and polyunsaturated FAs in 476 maternal serum samples at T1 and T3. A total of 36 FAs were analyzed although only 11 FAs were detected in serum of pregnant women and were summarized in Tables 2 and 3. The FA composition in maternal serum is represented as absolute (Table 2) and relative (Table 3) FA concentrations (mean $\pm \mathrm{SD}$ ) in both T1 and T3. The absolute total amount of FAs in maternal serum increased during pregnancy (T1, $10073.15 \mu \mathrm{mol} / \mathrm{L}$ and T3, 20,480.82 $\mu \mathrm{mol} / \mathrm{L}, p<0.01$ ) and the highest individual FAs were C16:0, C18:1n-9 and C18:2n-6 at both T1 and T3.

To compare the two reporting schemes, Pearson's correlations coefficients were calculated between FA values expressed in absolute and relative terms for the whole sample, which ranged from 464-476 serum samples, according to data available both in T1 and T3 of pregnancy (Supplementary Table S1). In T1, significant and positive correlations $(p<0.01)$ were observed for C12:0, C14:0, C16:0, C18:2n-6, C20:3n-6, C20:4n-6, C20:5n-3, C22:6n-6, $\Sigma$ total SFA, $\Sigma$ total n-6 PUFA and $\Sigma$ total n-3 PUFA. However, the Pearson's correlation coefficient was moderate $(\mathrm{r}<0.5)$ for the most of FAs in T1 with the exception of C16:0 $(\mathrm{r}=0.676), \mathrm{C} 20: 5 \mathrm{n}-3(\mathrm{r}=0.859)$ and $\Sigma$ total SFA $(\mathrm{r}=0.541)$ for which a strong correlation was observed. In contrast, non-significant correlations were observed for C18:0, C16:1n-7, C18:1n-9 and $\Sigma$ total MUFA. In T3, a positive correlation $(p<0.01)$ was observed for all FAs with the exception of C18:0 for which a moderate negative correlation $(p<0.01, \mathrm{r}=-0.387))$ was observed. In T3, the Pearson's correlation coefficient was low-moderate $(r=0.1-0.5)$ with the exception of C12:0 $(r=0.635), C 16: 0(r=0.724), C 20: 5 n-3(r=0.763)$ and $\Sigma$ total SFA $(r=0.605)$ for which a strong correlation was observed. At both sampling times T1 and $\mathrm{T} 3$, the FA with the highest strong positive correlation (T1, r = 0.859 and $\mathrm{T} 3, \mathrm{r}=0.763$ ) was C20:5n-3.

The corresponding percentiles of FAs during T1 and T3 were calculated and showed in Tables 2 and 3 and expressed as either an absolute concentration or a percentage of total FAs. Consequently, our reference ranges for relative concentration can be compared to the results expressed as mean \pm SD from other European studies $[14,15,19,38,39]$ shown in Table 4. The levels of individual FAs that were distinct from the 2.5 or 97.5 percentiles of the present study are indicated in Table 4 .

\subsection{Influence of Maternal Factors on Fatty Acid Serum Profiles}

Multiple linear regression models of the influence of maternal determinants on total FAs, total SFA, total MUFA, total n-6 PUFA, total n-3 PUFA, LA, DHA, and AA are shown in Table 5 . It can be observed that women with BMI $>30 \mathrm{~kg} / \mathrm{m}^{2}$ had significantly higher levels of total SFA, total MUFA and AA in both trimesters of pregnancy. Moreover, educational level and ethnicity were significantly associated with higher values of total n-3 PUFA and DHA in T1, and lower values of total SFA, total MUFA and AA in T3. In T1, only low physical activity was associated with lower values of DHA. However, DHA and total n-3 PUFA were significantly higher in T3 at better diet quality and older age. 
Table 2. Absolute concentration and percentile ( $\mu \mathrm{mol} / \mathrm{L})$ distribution of fatty acid in maternal serum during the first (T1) and third (T3) trimesters of pregnancy.

\begin{tabular}{|c|c|c|c|c|c|c|c|c|c|c|c|}
\hline \multirow[t]{2}{*}{ Fatty Acids } & & \multirow[t]{2}{*}{$\begin{array}{l}\text { Absolute Concentration } \\
(\mu \mathrm{mol} / \mathrm{L})^{*}\end{array}$} & \multicolumn{9}{|c|}{ Absolute Percentiles $(\mu \mathrm{mol} / \mathrm{L})$} \\
\hline & & & $\mathrm{P}_{2.5}$ & $\mathrm{P}_{5}$ & $\mathrm{P}_{10}$ & $\mathrm{P}_{25}$ & $\mathrm{P}_{50}$ & $\mathrm{P}_{75}$ & $\mathrm{P}_{90}$ & $\mathrm{P}_{95}$ & $\mathrm{P}_{97.5}$ \\
\hline \multicolumn{12}{|l|}{ SFA } \\
\hline \multirow[t]{2}{*}{ Lauric acid (C12:0) } & $\mathrm{T} 1, n=469$ & $40.14 \pm 10.50$ & 29.22 & 30.18 & 31.67 & 34.04 & 37.30 & 42.45 & 51.76 & 60.12 & 71.80 \\
\hline & $\mathrm{T} 3, n=466$ & $60.19 \pm 28.26$ & 32.52 & 34.51 & 36.73 & 40.91 & 51.10 & 69.71 & 97.49 & 121.81 & 146.67 \\
\hline \multirow{2}{*}{ Myristic acid (C14:0) } & $\mathrm{T} 1, n=471$ & $118.16 \pm 49.07$ & 57.95 & 63.99 & 69.33 & 83.20 & 107.42 & 139.15 & 188.35 & 228.58 & 260.84 \\
\hline & $\mathrm{T} 3, n=470$ & $207.80 \pm 80.31$ & 93.68 & 107.69 & 117.21 & 149.71 & 194.20 & 249.20 & 321.04 & 367.13 & 413.69 \\
\hline \multirow{2}{*}{ Palmitic acid (C16:0) } & $\mathrm{T} 1, n=467$ & $2904.40 \pm 1403.47$ & 1339.95 & 1463.74 & 1627.03 & 1937.08 & 2582.35 & 3500.79 & 4704.88 & 5573.20 & 7610.80 \\
\hline & $\mathrm{T} 3, n=466$ & $8511.32 \pm 4293.76$ & 2477.62 & 3017.91 & 3880.25 & 5507.61 & 7500.15 & $10,738.06$ & $14,966.15$ & $17,376.53$ & $18,980.26$ \\
\hline \multirow[t]{2}{*}{ Stearic acid (C18:0) } & $\mathrm{T} 1, n=470$ & $690.31 \pm 208.05$ & 398.5 & 435.26 & 465.17 & 547.46 & 639.97 & 804.96 & 1019.98 & 1139.45 & 1211.48 \\
\hline & $\mathrm{T} 3, n=472$ & $808.08 \pm 202.10$ & 474.67 & 522.63 & 569.76 & 664.25 & 779.99 & 930.30 & 1081.33 & 1174.58 & 1302.64 \\
\hline \multirow[t]{2}{*}{$\Sigma$ Total SFA } & $\mathrm{T} 1, n=467$ & $3765.31 \pm 1614.17$ & 1884.32 & 2073.77 & 2267.31 & 2646.97 & 3408.43 & 4504.30 & 5907.35 & 6961.68 & 8802.81 \\
\hline & $\mathrm{T} 3, n=467$ & $9625.65 \pm 4574.33$ & 3171.60 & 3756.01 & 4644.10 & 6401.81 & 8574.46 & $11,903.47$ & $16,488.0$ & $18,902.97$ & $20,791.60$ \\
\hline \multicolumn{12}{|l|}{ MUFA } \\
\hline \multirow{2}{*}{ Palmitoleic acid (C16:1n-7) } & $\mathrm{T} 1, n=465$ & $186.37 \pm 45.88$ & 119.70 & 125.68 & 136.35 & 151.80 & 180.84 & 211.59 & 250.73 & 283.54 & 296.39 \\
\hline & $\mathrm{T} 3, n=468$ & $256.66 \pm 89.61$ & 100.78 & 134.70 & 163.33 & 194.08 & 245.46 & 303.10 & 378.4 & 422.99 & 487.91 \\
\hline \multirow{2}{*}{ Oleic acid (C18:1n-9) } & $\mathrm{T} 1, n=466$ & $1444.74 \pm 461.90$ & 817.01 & 881.34 & 959.76 & 1097.08 & 1381.89 & 1666.88 & 2044.13 & 2333.50 & 2694.42 \\
\hline & $\mathrm{T} 3, n=466$ & $2843.85 \pm 1251.92$ & 1083.25 & 1319.01 & 1507.87 & 1915.78 & 2602.09 & 3514.33 & 4400.45 & 5507.76 & 6221.64 \\
\hline$\Sigma$ Total MUFA & $\mathrm{T} 1, n=466$ & $1634.54 \pm 500.21$ & 959.91 & 1020.19 & 1104.29 & 1257.50 & 1555.50 & 1900.07 & 2275.35 & 2596.12 & 2979.46 \\
\hline \multicolumn{12}{|l|}{ n-6 PUFA } \\
\hline \multirow[t]{2}{*}{ LA (C18:2n-6) } & $\mathrm{T} 1, n=466$ & $3355.67 \pm 1230.19$ & 1602.64 & 1756.72 & 1927.14 & 2439.91 & 3213.76 & 4097.77 & 5012.97 & 5710.44 & 6211.58 \\
\hline & $\mathrm{T} 3, n=470$ & $6321.14 \pm 2786.02$ & 2408.12 & 2737.37 & 3282.10 & 4194.24 & 5722.28 & 7957.48 & $10,332.80$ & $11,602.18$ & $13,327.69$ \\
\hline \multirow{2}{*}{ DHGLA (C20:3n-6) } & $\mathrm{T} 1, n=469$ & $229.99 \pm 90.64$ & 101.13 & 114.98 & 127.81 & 165.93 & 216.25 & 280.67 & 360.30 & 407.91 & 450.08 \\
\hline & $\mathrm{T} 3, n=471$ & $246.20 \pm 85.27$ & 116.31 & 129.41 & 146.81 & 184.03 & 233.43 & 300.70 & 363.36 & 397.26 & 445.50 \\
\hline \multirow{2}{*}{ AA (C20:4n-6) } & $\mathrm{T} 1, n=473$ & $830.82 \pm 276.15$ & 421.52 & 455.60 & 520.47 & 631.14 & 791.72 & 991.31 & 1247.92 & 1398.0 & 1523.56 \\
\hline & T3, $n=469$ & $722.63 \pm 220.05$ & 387.52 & 423.29 & 472.62 & 560.47 & 692.0 & 855.29 & 1035.63 & 1114.09 & 1223.84 \\
\hline \multirow[t]{2}{*}{$\Sigma$ Total n-6 PUFA } & $\mathrm{T} 1, n=465$ & $4433.77 \pm 1469.64$ & 2325.77 & 2420.11 & 2697.52 & 3325.21 & 4219.65 & 5333.68 & 6482.27 & 7114.28 & 7735.74 \\
\hline & $\mathrm{T} 3, n=469$ & $7278.06 \pm 2919.04$ & 3126.65 & 3435.44 & 4072.94 & 5029.21 & 6758.36 & 9100.77 & $11,346.52$ & $12,917.44$ & $14,348.43$ \\
\hline \multicolumn{12}{|l|}{ n-3 PUFA } \\
\hline \multirow[t]{2}{*}{ EPA (C20:5n-3) } & $\mathrm{T} 1, n=467$ & $35.03 \pm 23.95$ & 7.80 & 9.51 & 11.58 & 17.67 & 29.30 & 45.36 & 74.50 & 87.17 & 103.34 \\
\hline & $\mathrm{T} 3, n=470$ & $23.88 \pm 16.93$ & 3.96 & 5.07 & 6.35 & 11.10 & 19.04 & 32.31 & 47.78 & 59.45 & 68.46 \\
\hline \multirow[t]{2}{*}{ DHA (C22:6n-3) } & $\mathrm{T} 1, n=473$ & $240.28 \pm 73.47$ & 119.02 & 134.43 & 151.61 & 185.65 & 234.05 & 291.00 & 340.30 & 381.36 & 406.58 \\
\hline & $\mathrm{T} 3, n=474$ & $236.60 \pm 71.77$ & 123.02 & 132.54 & 150.80 & 182.27 & 224.54 & 283.39 & 336.38 & 374.51 & 396.16 \\
\hline \multirow{2}{*}{$\Sigma$ Total n-3 PUFA } & $\mathrm{T} 1, n=473$ & $276.98 \pm 94.07$ & 129.01 & 149.88 & 169.46 & 205.28 & 264.05 & 332.67 & 414.77 & 469.19 & 495.58 \\
\hline & $\mathrm{T} 3, n=473$ & $260.52 \pm 84.33$ & 133.12 & 141.08 & 160.92 & 194.55 & 250.85 & 315.61 & 377.51 & 426.69 & 454.23 \\
\hline
\end{tabular}


Table 2. Cont

\begin{tabular}{|c|c|c|c|c|c|c|c|c|c|c|c|}
\hline \multicolumn{2}{|c|}{ Fatty Acids } & \multirow{2}{*}{$\begin{array}{l}\text { Absolute Concentration } \\
(\mu \mathrm{mol} / \mathrm{L})^{*}\end{array}$} & \multicolumn{9}{|c|}{ Absolute Percentiles $(\mu \mathrm{mol} / \mathrm{L})$} \\
\hline & & & $\mathrm{P}_{2.5}$ & $\mathrm{P}_{5}$ & $\mathrm{P}_{10}$ & $\mathrm{P}_{25}$ & $\mathrm{P}_{50}$ & $\mathrm{P}_{75}$ & $\mathrm{P}_{90}$ & $\mathrm{P}_{95}$ & $\mathrm{P}_{97.5}$ \\
\hline Total FAs & $\mathrm{T} 1, n=464$ & $10,073.15 \pm 3349.07$ & 5589.89 & 5808.29 & 6420.65 & 7561.30 & 9551.76 & $11,982.98$ & $14,557.61$ & $16,156.53$ & $18,491.35$ \\
\hline \multirow[t]{2}{*}{$n-6 / n-3$ ratio ${ }^{(a)}$} & $\mathrm{T} 1, n=471$ & $16.80 \pm 5.19$ & 8.39 & 9.25 & 10.54 & 13.23 & 16.39 & 19.70 & 24.25 & 27.01 & 29.61 \\
\hline & $\mathrm{T} 3, n=471$ & $29.29 \pm 11.52$ & 11.83 & 13.44 & 15.77 & 20.98 & 27.69 & 36.54 & 46.83 & 51.60 & 54.88 \\
\hline \multirow[t]{2}{*}{ AA/EPA ratio } & $\mathrm{T} 1, n=469$ & $31.77 \pm 19.08$ & 6.31 & 7.66 & 11.10 & 17.73 & 27.44 & 42.68 & 57.56 & 72.19 & 80.34 \\
\hline & $\mathrm{T} 3, n=470$ & $44.09 \pm 30.61$ & 9.19 & 10.80 & 13.36 & 22.56 & 37.83 & 57.30 & 92.27 & 113.43 & 133.61 \\
\hline \multirow[t]{2}{*}{$\mathrm{AA} /(\mathrm{EPA}+\mathrm{DHA})$} & $\mathrm{T} 1, n=474$ & $3.12 \pm 0.87$ & 1.65 & 1.83 & 2.05 & 2.49 & 3.05 & 3.73 & 4.32 & 4.53 & 5.06 \\
\hline & $\mathrm{T} 3, n=475$ & $2.96 \pm 0.91$ & 1.46 & 1.61 & 1.82 & 2.30 & 2.85 & 3.56 & 4.14 & 4.55 & 4.99 \\
\hline \multirow[t]{2}{*}{ LA/DHGLA ratio } & $\mathrm{T} 1, n=469$ & $15.45 \pm 5.12$ & 7.40 & 8.03 & 9.67 & 11.84 & 14.80 & 18.55 & 23.20 & 25.04 & 27.86 \\
\hline & $\mathrm{T} 3, n=471$ & $26.59 \pm 10.58$ & 11.59 & 12.97 & 14.44 & 18.71 & 24.20 & 32.78 & 42.46 & 47.05 & 51.60 \\
\hline \multirow[t]{2}{*}{ EFA index } & $\mathrm{T} 1, n=473$ & $2.91 \pm 0.64$ & 1.77 & 1.94 & 2.11 & 2.49 & 2.85 & 3.30 & 3.74 & 4.05 & 4.37 \\
\hline & $\mathrm{T} 3, n=470$ & $2.53 \pm 0.90$ & 1.20 & 1.30 & 1.48 & 1.90 & 2.39 & 3.07 & 3.81 & 4.29 & 4.71 \\
\hline
\end{tabular}

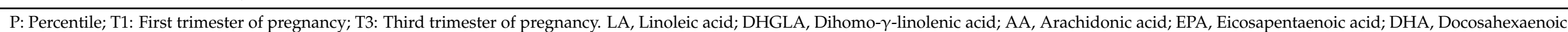

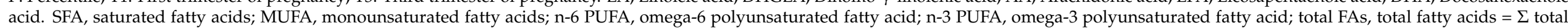

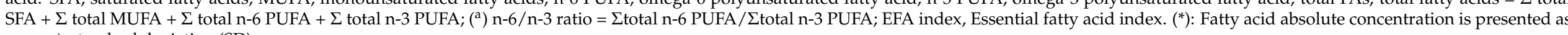
mean \pm standard deviation $(\mathrm{SD})$

Table 3. Relative concentration and percentile (\%) distribution of fatty acid in maternal serum during the first (T1) and third (T3) trimester of pregnancy.

\begin{tabular}{|c|c|c|c|c|c|c|c|c|c|c|c|}
\hline \multirow{2}{*}{\multicolumn{2}{|c|}{ Fatty Acids }} & \multirow{2}{*}{$\begin{array}{l}\text { Relative Concentration } \\
\text { (\% of Total FAs) }\end{array}$} & \multicolumn{9}{|c|}{ Relative Percentiles (\%) } \\
\hline & & & $\mathrm{P}_{2.5}$ & $\mathrm{P}_{5}$ & $\mathrm{P}_{10}$ & $\mathrm{P}_{25}$ & $\mathrm{P}_{50}$ & $\mathrm{P}_{75}$ & $\mathrm{P}_{90}$ & $\mathrm{P}_{95}$ & $\mathrm{P}_{97.5}$ \\
\hline \multicolumn{12}{|l|}{ SFA } \\
\hline \multirow[t]{2}{*}{ Lauric acid (C12:0) } & $\mathrm{T} 1, n=472$ & $0.42 \pm 0.13$ & 0.20 & 0.23 & 0.27 & 0.33 & 0.40 & 0.49 & 0.60 & 0.65 & 0.74 \\
\hline & $\mathrm{T} 3, n=470$ & $0.34 \pm 0.20$ & 0.12 & 0.14 & 0.16 & 0.20 & 0.28 & 0.40 & 0.62 & 0.79 & 0.94 \\
\hline \multirow[t]{2}{*}{ Myristic acid (C14:0) } & $\mathrm{T} 1, n=472$ & $1.17 \pm 0.32$ & 0.65 & 0.71 & 0.80 & 0.94 & 1.12 & 1.37 & 1.62 & 1.74 & 1.90 \\
\hline & $\mathrm{T} 3, n=473$ & $1.07 \pm 0.36$ & 0.49 & 0.56 & 0.67 & 0.81 & 1.02 & 1.29 & 1.59 & 1.76 & 1.97 \\
\hline Palmitic acid (C16:0) & $\mathrm{T} 3, n=475$ & $40.89 \pm 6.82$ & 28.06 & 30.81 & 32.88 & 36.17 & 40.47 & 44.98 & 49.74 & 52.76 & 57.08 \\
\hline Stearic acid (C18:0) & $\mathrm{T} 1, n=475$ & $6.88 \pm 1.06$ & 4.41 & 4.41 & 4.90 & 5.42 & 6.23 & 7.01 & 7.64 & 8.14 & 8.47 \\
\hline
\end{tabular}


Table 3. Cont

\begin{tabular}{|c|c|c|c|c|c|c|c|c|c|c|c|}
\hline \multirow[t]{2}{*}{ Fatty Acids } & & \multirow[t]{2}{*}{$\begin{array}{l}\text { Relative Concentration } \\
\quad(\% \text { of Total FAs) }\end{array}$} & \multicolumn{9}{|c|}{ Relative Percentiles (\%) } \\
\hline & & & $\mathrm{P}_{2.5}$ & $\mathrm{P}_{5}$ & $\mathrm{P}_{10}$ & $\mathrm{P}_{25}$ & $\mathrm{P}_{50}$ & $\mathrm{P}_{75}$ & $\mathrm{P}_{90}$ & $\mathrm{P}_{95}$ & $\mathrm{P}_{97.5}$ \\
\hline \multirow{3}{*}{$\Sigma$ Total SFA } & $\mathrm{T} 3, n=474$ & $4.20 \pm 1.04$ & 2.49 & 2.65 & 2.91 & 3.48 & 4.09 & 4.81 & 5.57 & 6.27 & 6.76 \\
\hline & $\mathrm{T} 1, n=470$ & $36.57 \pm 4.38$ & 28.84 & 30.01 & 30.59 & 33.49 & 36.55 & 39.34 & 42.02 & 44.15 & 45.65 \\
\hline & $\mathrm{T} 3, n=474$ & $46.51 \pm 6.27$ & 35.89 & 37.41 & 39.08 & 42.10 & 46.06 & 50.42 & 54.30 & 58.07 & 62.08 \\
\hline \multicolumn{12}{|l|}{ MUFA } \\
\hline Palmitoleic acid (C16:1n-7) & $\mathrm{T} 1, n=475$ & $1.92 \pm 0.45$ & 1.14 & 1.25 & 1.39 & 1.62 & 1.87 & 2.19 & 2.58 & 2.78 & 2.96 \\
\hline \multirow[t]{2}{*}{ Oleic acid (C18:1n-9) } & $\mathrm{T} 1, n=474$ & $14.52 \pm 2.27$ & 10.22 & 11.18 & 11.89 & 13.02 & 14.24 & 16.10 & 17.68 & 18.57 & 19.50 \\
\hline & $\mathrm{T} 3, n=471$ & $14.16 \pm 3.36$ & 8.52 & 9.11 & 10.13 & 11.68 & 13.72 & 16.37 & 18.77 & 20.32 & 21.40 \\
\hline \multirow[t]{2}{*}{$\Sigma$ Total MUFA } & $\mathrm{T} 1, n=475$ & $16.46 \pm 2.53$ & 11.47 & 12.57 & 13.55 & 14.79 & 16.18 & 18.16 & 19.83 & 20.91 & 21.68 \\
\hline & $\mathrm{T} 3, n=473$ & $15.58 \pm 3.51$ & 9.50 & 10.49 & 11.32 & 12.93 & 15.14 & 17.84 & 20.19 & 31.81 & 22.98 \\
\hline \multicolumn{12}{|l|}{ n-6 PUFA } \\
\hline \multirow[t]{2}{*}{ LA (C18:2n-6), $n=446$} & $\mathrm{~T} 1, n=476$ & $33.30 \pm 5.19$ & 23.21 & 24.90 & 26.58 & 29.82 & 33.38 & 36.81 & 39.95 & 42.13 & 44.14 \\
\hline & $\mathrm{T} 3, n=476$ & $31.13 \pm 6.95$ & 17.65 & 19.97 & 22.21 & 26.28 & 31.03 & 35.61 & 40.23 & 42.68 & 45.69 \\
\hline DHGLA (C20:3n-6), $n=450$ & $\mathrm{~T} 1, n=473$ & $2.26 \pm 0.58$ & 1.20 & 1.38 & 1.54 & 1.84 & 2.21 & 2.61 & 2.96 & 3.33 & 3.52 \\
\hline \multirow[t]{2}{*}{ AA $(C 20: 4 n-6), n=451$} & $\mathrm{~T} 1, n=475$ & $8.31 \pm 2.05$ & 4.33 & 4.88 & 5.62 & 7.09 & 8.29 & 9.66 & 10.95 & 11.89 & 12.69 \\
\hline & $\mathrm{T} 3, n=471$ & $3.83 \pm 1.33$ & 1.79 & 2.03 & 2.27 & 2.91 & 3.62 & 4.54 & 5.69 & 6.30 & 7.55 \\
\hline \multirow[t]{2}{*}{$\Sigma$ Total n-6 PUFA, $n=444$} & $\mathrm{~T} 1, n=474$ & $44.35 \pm 5.07$ & 33.62 & 36.25 & 38.20 & 41.05 & 44.59 & 47.44 & 50.80 & 52.94 & 54.46 \\
\hline & $\mathrm{T} 3, n=476$ & $36.50 \pm 7.27$ & 21.78 & 24.07 & 27.36 & 31.43 & 36.47 & 40.95 & 45.85 & 48.62 & 50.86 \\
\hline \multicolumn{12}{|l|}{ n-3 PUFA } \\
\hline \multirow[t]{2}{*}{ EPA (C20:5n-3), $n=446$} & $\mathrm{~T} 1, n=467$ & $0.36 \pm 0.24$ & 0.07 & 0.11 & 0.13 & 0.18 & 0.30 & 0.46 & 0.66 & 0.86 & 1.04 \\
\hline & $\mathrm{T} 3, n=470$ & $0.13 \pm 0.10$ & 0.02 & 0.03 & 0.04 & 0.06 & 0.10 & 0.16 & 0.27 & 0.33 & 0.39 \\
\hline \multirow[t]{2}{*}{ DHA (C22:6n-3), $n=456$} & $\mathrm{~T} 1, n=474$ & $2.24 \pm 0.66$ & 1.25 & 1.49 & 1.63 & 1.97 & 2.37 & 2.85 & 3.35 & 3.58 & 3.87 \\
\hline & $\mathrm{T} 3, n=472$ & $1.24 \pm 0.46$ & 0.58 & 0.65 & 0.73 & 0.92 & 1.17 & 1.50 & 1.89 & 2.08 & 2.41 \\
\hline \multirow[b]{2}{*}{$\Sigma$ Total n-3 PUFA } & $\mathrm{T} 1, n=470$ & $2.77 \pm 0.83$ & 1.41 & 1.62 & 1.79 & 2.16 & 2.67 & 3.28 & 3.93 & 4.35 & 4.74 \\
\hline & $\mathrm{T} 3, n=470$ & $1.36 \pm 0.53$ & 0.64 & 0.70 & 0.78 & 0.98 & 1.27 & 1.66 & 2.12 & 2.42 & 2.67 \\
\hline \multirow[t]{2}{*}{$n-6 / n-3$ ratio $^{(a)}$} & $\mathrm{T} 1, n=472$ & $16.93 \pm 5.27$ & 8.39 & 9.25 & 10.54 & 13.23 & 16.40 & 19.70 & 24.41 & 27.53 & 29.67 \\
\hline & $\mathrm{T} 3, n=472$ & $29.54 \pm 11.66$ & 11.83 & 13.45 & 15.78 & 21.02 & 27.71 & 36.63 & 46.99 & 51.68 & 55.53 \\
\hline
\end{tabular}


Table 3. Cont

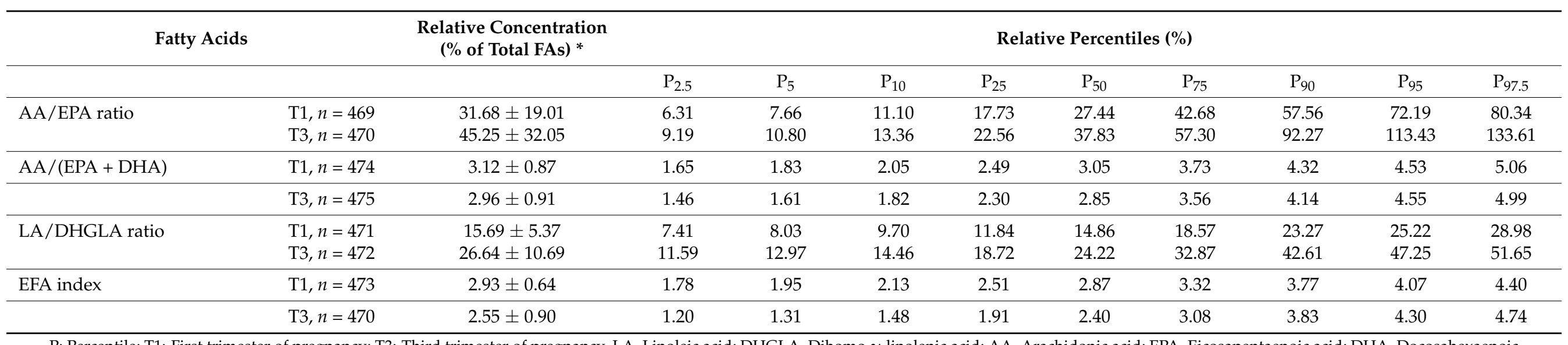

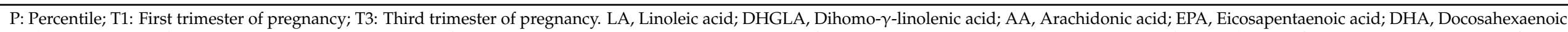

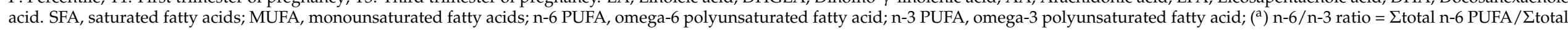
n-3 PUFA; EFA index, Essential fatty acid index. $\left(^{*}\right)$ : Fatty acid relative concentration is presented as mean \pm standard deviation (SD).

Table 4. A comparison of reported concentrations (\%) of total fatty acids in maternal serum from different European countries

\begin{tabular}{|c|c|c|c|c|c|c|c|}
\hline \multicolumn{2}{|l|}{ Fatty Acids } & \multirow{2}{*}{$\begin{array}{c}\begin{array}{c}\text { Our Reference Intervals } \\
(\mathrm{T} 1, n=472 ; \mathrm{T} 3, n=468)\end{array} \\
\text { Percentiles }(2.5-97.5 \%)\end{array}$} & \multirow[t]{2}{*}{$\begin{array}{l}\text { Montes et al. [19] }(n=170) \\
\text { (Spain) }\end{array}$} & \multirow[t]{2}{*}{$\begin{array}{l}\text { Otto et al. [14] }(n=23) \\
\text { (The Netherlands) }\end{array}$} & \multirow{2}{*}{$\begin{array}{c}\begin{array}{c}\text { Enke et al. [15] }(\boldsymbol{n}=\mathbf{5 5}) \\
\text { (Germany) }\end{array} \\
\text { Mean } \pm \text { SD } \\
\end{array}$} & \multirow[t]{2}{*}{$\begin{array}{c}\text { Vlaardingerbroek et al. } \\
\text { [38] }(n=172) \\
\text { (The Netherlands) }\end{array}$} & \multirow[t]{2}{*}{$\begin{array}{l}\text { Wheeler et al. [39] ( } n=142) \\
\text { (United Kingdom) }\end{array}$} \\
\hline & & & & & & & \\
\hline \multicolumn{8}{|l|}{ SFA } \\
\hline \multirow{2}{*}{ Myristic acid (C14:0) } & $\mathrm{T} 1$ & $0.65-1.90$ & - & - & - & - & \\
\hline & T3 & $0.49-1.97$ & - & - & $1.21 \pm 0.40$ & - & $1.21 \pm 0.03$ \\
\hline \multirow[t]{2}{*}{ Palmitic acid (C16:0) } & $\mathrm{T} 1$ & $20.29-38.10$ & - & - & - & - & \\
\hline & T3 & $28.06-57.08$ & - & - & $30.20 \pm 2.31 *$ & - & $25.9 \pm 0.17 *$ \\
\hline \multirow[t]{2}{*}{ Stearic acid (C18:0) } & $\mathrm{T} 1$ & $4.41-8.47$ & - & - & - & - & \\
\hline & $\mathrm{T} 3$ & $2.49-6.76$ & - & - & $5.59 \pm 0.78$ & - & $5.84 \pm 0.05$ \\
\hline \multirow[t]{2}{*}{$\Sigma$ Total SFA } & $\mathrm{T} 1$ & $28.84-45.65$ & $30.34 \pm 2.06$ & $44.14 \pm 0.46$ & - & $45.06 \pm 0.09$ & \\
\hline & $\mathrm{T} 3$ & $25.89-62.08$ & - & - & $67.80 \pm 4.77 \ddagger$ & $45.80 \pm 0.07$ & $33.0 \pm 0.20$ \\
\hline \multicolumn{8}{|l|}{ MUFA } \\
\hline \multirow{2}{*}{ Palmitoleic acid (C16:1n-7) } & $\mathrm{T} 1$ & $1.14-2.96$ & - & - & - & - & \\
\hline & $\mathrm{T} 3$ & $0.61-2.52$ & - & - & - & - & $2.41 \pm 0.07$ \\
\hline
\end{tabular}


Table 4. Cont

\begin{tabular}{|c|c|c|c|c|c|c|c|}
\hline Fatty Acids & & $\begin{array}{l}\text { Our Reference Intervals } \\
(\mathrm{T} 1, n=472 ; \mathrm{T} 3, n=468)\end{array}$ & $\begin{array}{c}\text { Montes et al. [19] }(n=170) \\
\text { (Spain) }\end{array}$ & $\begin{array}{l}\text { Otto et al. [14] }(n=23) \\
\text { (The Netherlands) }\end{array}$ & $\begin{array}{c}\text { Enke et al. [15] }(n=55) \\
\text { (Germany) }\end{array}$ & $\begin{array}{c}\text { Vlaardingerbroek et al. } \\
{[38](n=172)} \\
\text { (The Netherlands) }\end{array}$ & $\begin{array}{l}\text { Wheeler et al. [39] }(n=142) \\
\quad \text { (United Kingdom) }\end{array}$ \\
\hline & & Percentiles (2.5-97.5\%) & & & Mean \pm SD & & \\
\hline Oleic acid (C18:1n-9) & $\mathrm{T} 3$ & $8.52-21.40$ & - & - & $23.90 \pm 2.45 \ddagger$ & - & $22.2 \pm 0.40 \ddagger$ \\
\hline \multirow[t]{2}{*}{$\Sigma$ Total MUFA } & $\mathrm{T} 1$ & $11.47-21.68$ & $23.29 \pm 3.62 \ddagger$ & $12.05 \pm 0.25$ & - & $11.20 \pm 0.08^{*}$ & \\
\hline & $\mathrm{T} 3$ & $9.50-22.98$ & - & - & $29.20 \pm 2.93 \ddagger$ & $11.99 \pm 0.10$ & $28.8 \pm 0.25^{\ddagger}$ \\
\hline \multicolumn{8}{|l|}{ n-6 PUFA } \\
\hline \multirow[t]{2}{*}{ LA (C18:2n-6) } & $\mathrm{T} 1$ & $23.21-44.14$ & $32.16 \pm 4.16$ & $20.48 \pm 0.48 *$ & - & $21.28 \pm 0.18 *$ & \\
\hline & $\mathrm{T} 3$ & $17.65-45.69$ & - & - & $23.50 \pm 3.41$ & $21.78 \pm 0.18$ & $27.1 \pm 0.29$ \\
\hline \multirow[t]{2}{*}{ DHGLA (C20:3n-6) } & $\mathrm{T} 1$ & $1.20-3.52$ & $1.55 \pm 0.56^{*}$ & $3.53 \pm 0.17 \ddagger$ & - & $3.22 \pm 0.05$ & \\
\hline & $\mathrm{T} 3$ & $0.63-2.20$ & - & - & $1.55 \pm 0.32$ & $3.46 \pm 0.04 ¥$ & $7.88 \pm 0.20 \nRightarrow$ \\
\hline \multirow[t]{2}{*}{ AA (C20:4n-6) } & $\mathrm{T} 1$ & $4.33-12.69$ & $7.33 \pm 1.74$ & $9.34 \pm 0.35$ & - & $9.64 \pm 0.11$ & \\
\hline & T3 & $1.79-7.55$ & - & - & $3.83 \pm 0.89$ & $8.15 \pm 0.10^{\ddagger}$ & $5.40 \pm 0.08$ \\
\hline \multirow[t]{2}{*}{$\Sigma$ Total n-6 PUFA } & $\mathrm{T} 1$ & $33.62-54.46$ & $42.56 \pm 4.22$ & $34.88 \pm 0.62$ & - & - & \\
\hline & T3 & $21.78-50.86$ & - & - & - & - & $34.7 \pm 0.31$ \\
\hline \multicolumn{8}{|l|}{ n-3 PUFA } \\
\hline EPA (C20:5n-3) & T3 & $0.02-0.39$ & - & - & $0.24 \pm 0.10$ & $0.34 \pm 0.02$ & $0.32 \pm 0.02$ \\
\hline \multirow[t]{2}{*}{ DHA (C22:6n-3) } & $\mathrm{T} 1$ & $1.25-3.87$ & $2.68 \pm 0.62$ & $3.93 \pm 0.22 \ddagger$ & - & $3.88 \pm 0.07^{\sharp}$ & \\
\hline & $\mathrm{T} 3$ & $0.58-2.41$ & - & - & $1.21 \pm 0.35$ & $3.74 \pm 0.06^{\ddagger}$ & $2.07 \pm 0.04$ \\
\hline \multirow{2}{*}{$\Sigma$ Total n-3 PUFA } & $\mathrm{T} 1$ & $1.41-4.74$ & $3.69 \pm 0.87$ & $5.35 \pm 0.33 \ddagger$ & - & - & \\
\hline & $\mathrm{T} 3$ & $0.64-2.67$ & - & - & $1.61 \pm 0.46$ & - & $2.72 \pm 0.06^{\ddagger}$ \\
\hline \multirow[t]{2}{*}{ n-6/n-3 ratio ${ }^{(a)}$} & $\mathrm{T} 1$ & $8.39-29.61$ & $12.16 \pm 3.04$ & $6.44 \pm 0.29 *$ & - & - & \\
\hline & $\mathrm{T} 3$ & $11.83-54.88$ & - & - & - & - & \\
\hline \multirow[t]{2}{*}{$\mathrm{AA} /(\mathrm{EPA}+\mathrm{DHA})$} & $\mathrm{T} 1$ & $1.65-5.06$ & - & - & - & - & \\
\hline & $\mathrm{T} 3$ & $1.46-4.99$ & - & - & - & - & $2.32 \pm 0.04$ \\
\hline \multirow[t]{2}{*}{ EFA index } & T1 & $1.78-4.40$ & - & $3.48 \pm 0.09$ & - & $3.62 \pm 0.04$ & \\
\hline & T3 & $1.20-4.74$ & - & - & - & $3.34 \pm 0.04$ & \\
\hline
\end{tabular}

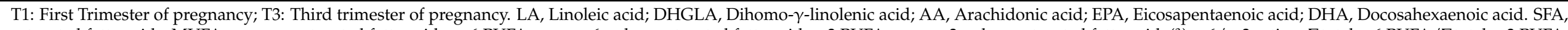

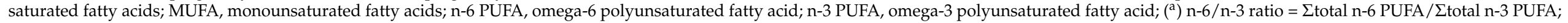

EFA index, Essential fatty acid index. (-) indicates that the fatty acid is not reported. The $\left(^{*}\right)$ and $\left({ }^{\ddagger}\right)$ denote: under 2.5 percentile and over 97.5 percentile of the present study, respectively. 
Table 5. Multiple linear regression of potential factors related to maternal serum fatty acids composition in the first (T1) and third (T3) trimesters of pregnancy.

\begin{tabular}{|c|c|c|c|c|c|c|c|c|c|c|c|c|}
\hline \multicolumn{13}{|c|}{ Fatty Acids } \\
\hline & \multicolumn{3}{|c|}{ Total FA } & \multicolumn{3}{|c|}{ Total SFA } & \multicolumn{3}{|c|}{ Total MUFA } & \multicolumn{3}{|c|}{ Total n-6 PUFA } \\
\hline Maternal factors in $\mathrm{T} 1$ & B & SE & $p$ & B & SE & $p$ & $\mathrm{~B}$ & SE & $p$ & B & SE & $p$ \\
\hline Maternal Age & 5.8 & 44.0 & 0.895 & -14.5 & 21.1 & 0.494 & 0.3 & 6.6 & 0.959 & 2.1 & 19.6 & 0.915 \\
\hline Occupation & 100.9 & 423.8 & 0.812 & 115.2 & 202.1 & 0.569 & 32.3 & 63.8 & 0.614 & 22.2 & 188.3 & 0.906 \\
\hline Educational level & -152.8 & 299.0 & 0.610 & 16.2 & 143.1 & 0.910 & 47.1 & 45.4 & 0.300 & -124.4 & 132.8 & 0.350 \\
\hline Ethnicity & 395.4 & 578.6 & 0.495 & 334.0 & 275.5 & 0.226 & 108.4 & 86.4 & 0.211 & 93.5 & 256.8 & 0.716 \\
\hline Parity & 403.7 & 412.6 & 0.329 & 417.3 & 198.2 & $0.036 *$ & 116.5 & 62.4 & 0.063 & 115.2 & 183.0 & 0.530 \\
\hline BMI & 97.3 & 43.2 & 0.025 & 56.1 & 20.4 & $0.006 *$ & 13.2 & 6.5 & 0.044 * & 34.7 & 19.2 & 0.072 \\
\hline Gestational weight gain & 49.8 & 48.3 & 0.304 & -27.5 & 23.1 & 0.236 & -0.1 & 7.3 & 0.992 & -19.9 & 21.5 & 0.354 \\
\hline Alcohol consumption & -2790.3 & 3316.8 & 0.401 & -1917.9 & 1589.0 & 0.228 & -567.4 & 502.5 & 0.260 & -468.6 & 1473.4 & 0.751 \\
\hline Physical Activity & -145.6 & 333.0 & 0.662 & 6.4 & 159.4 & 0.968 & -25.2 & 50.4 & 0.617 & -118.3 & 147.8 & 0.424 \\
\hline \multirow[t]{2}{*}{ Diet Quality } & 106.3 & 73.4 & 0.149 & 55.8 & 35.1 & 0.113 & 16.5 & 11.1 & 0.138 & 21.0 & 32.6 & 0.520 \\
\hline & \multicolumn{3}{|c|}{$\mathrm{R}^{2}=0.056, \mathrm{~F}_{11,280}=1.502 ; p=0.130$} & \multicolumn{3}{|c|}{$\mathrm{R} 2=0.081, \mathrm{~F}_{11,281}=2.248 ; p=0.012 *$} & \multicolumn{3}{|c|}{$\mathrm{R}^{2}=0.060, \mathrm{~F}_{11,281}=1.632 ; p=0.089$} & \multicolumn{3}{|c|}{$\mathrm{R}^{2}=0.041, \mathrm{~F}_{11,281}=1.101 ; p=0.360$} \\
\hline \multicolumn{13}{|l|}{ Maternal factors in T3 } \\
\hline Maternal Age & 191.2 & 108.3 & 0.079 & 37.0 & 59.8 & 0.536 & 37.2 & 17.9 & 0.080 & 36.5 & 38.2 & 0.340 \\
\hline Occupation & -1059.6 & 1058.4 & 0.318 & -878.9 & 576.9 & 0.129 & -306.2 & 174.3 & 0.511 & 154.0 & 369.9 & 0.677 \\
\hline Educational level & -1453.6 & 737.6 & 0.050 & -1002.6 & 406.6 & 0.014 * & -81.1 & 123.4 & $0.026^{*}$ & -446.0 & 259.0 & 0.086 \\
\hline Ethnicity & -1851.1 & 1429.2 & 0.196 & -922.6 & 785.1 & 0.241 & -527.1 & 235.1 & 0.384 & -761.8 & 500.1 & 0.129 \\
\hline Parity & -745.5 & 1030.0 & 0.470 & 739.1 & 569.5 & 0.195 & -148.1 & 170.0 & 0.014 * & -483.3 & 360.8 & 0.181 \\
\hline Gestational weight gain & 149.2 & 119.7 & 0.214 & 107.2 & 66.2 & 0.106 & 9.5 & 20.0 & 0.229 & 17.3 & 42.2 & 0.683 \\
\hline Smoking & -271.5 & 638.2 & 0.671 & -386.9 & 354.1 & 0.275 & -127.9 & 106.1 & 0.659 & -36.2 & 223.8 & 0.872 \\
\hline Alcohol consumption & -4228.6 & 8281.3 & 0.610 & -1697.3 & 4543.5 & 0.709 & -609.0 & 1378.4 & 0.475 & -2083.2 & 2909.4 & 0.475 \\
\hline Physical Activity & -482.1 & 818.8 & 0.557 & -688.4 & 456.4 & 0.133 & -97.2 & 136.0 & 0.507 & -49.0 & 287.8 & 0.865 \\
\hline \multirow[t]{3}{*}{ Diet Quality } & 130.1 & 182.9 & 0.477 & 89.0 & 100.2 & 0.375 & 20.2 & 30.3 & 0.080 & 58.8 & 64.5 & 0.363 \\
\hline & \multicolumn{3}{|c|}{$\mathrm{R}^{2}=0.061, \mathrm{~F}_{11,283}=1.673 ; p=0.079$} & \multicolumn{3}{|c|}{$\mathrm{R}^{2}=0.091, \mathrm{~F}_{11,282}=2.573 ; p=0.004^{*}$} & \multicolumn{3}{|c|}{$\mathrm{R}^{2}=0.091, \mathrm{~F}_{11,285}=2.587 ; p=0.004^{*}$} & \multicolumn{3}{|c|}{$\mathrm{R}^{2}=0.041, \mathrm{~F}_{11,284}=1.098 ; p=0.362$} \\
\hline & \multicolumn{3}{|c|}{ Total n-3 PUFA } & \multicolumn{3}{|c|}{ Linoleic Acid } & \multicolumn{3}{|c|}{ Arachidonic Acid } & \multicolumn{3}{|c|}{ Docosahexaenoic Acid } \\
\hline Maternal factors in T1 & $\mathrm{B}$ & SE & $p$ & B & SE & $p$ & B & SE & $p$ & $\mathrm{~B}$ & SE & $p$ \\
\hline Maternal Age & 2.9 & 1.3 & $0.027 *$ & 5.6 & 16.5 & 0.735 & -1.6 & 3.7 & 0.671 & 1.6 & 1.0 & 0.101 \\
\hline Occupation & -9.1 & 12.5 & 0.465 & -52.1 & 159.1 & 0.744 & 26.7 & 36.1 & 0.460 & -9.7 & 9.7 & 0.316 \\
\hline Educational level & 23.3 & 8.8 & $0.009 *$ & -161.1 & 111.8 & 0.151 & 33.4 & 25.6 & 0.193 & 17.2 & 6.8 & $0.012 *$ \\
\hline Ethnicity & 42.3 & 16.8 & 0.012 * & 11.5 & 216.7 & 0.958 & 6.8 & 48.6 & 0.889 & 29.1 & 13.0 & $0.026^{*}$ \\
\hline Parity & 13.8 & 12.1 & 0.257 & 81.4 & 154.6 & 0.599 & 70.2 & 35.1 & $0.046^{*}$ & 11.2 & 9.3 & 0.233 \\
\hline BMI & 0.7 & 1.3 & 0.600 & 29.0 & 16.2 & 0.074 & 8.6 & 3.6 & 0.018 * & 0.8 & 1.0 & 0.404 \\
\hline Gestational weight gain & -0.2 & 1.4 & 0.880 & -16.0 & 18.1 & 0.379 & 0.3 & 4.1 & 0.950 & -0.2 & 1.1 & 0.834 \\
\hline Smoking & -2.6 & 7.6 & 0.736 & -122.6 & 96.1 & 0.203 & 8.2 & 22.0 & 0.709 & -3.3 & 5.8 & 0.570 \\
\hline Alcohol consumption & -67.8 & 97.8 & 0.489 & -156.9 & 1245.0 & 0.900 & -303.4 & 284.6 & 0.287 & -25.1 & 75.6 & 0.740 \\
\hline Physical Activity & -17.9 & 9.8 & 0.068 & -30.2 & 124.9 & 0.809 & -64.7 & 28.4 & 0.024 & -17.4 & 7.6 & $0.023 *$ \\
\hline
\end{tabular}


Table 5. Cont.

\begin{tabular}{|c|c|c|c|c|c|c|c|c|c|c|c|c|}
\hline \multicolumn{13}{|c|}{ Fatty Acids } \\
\hline \multirow[b]{2}{*}{ Diet Quality } & \multicolumn{3}{|c|}{ Total FA } & \multicolumn{3}{|c|}{ Total SFA } & \multicolumn{3}{|c|}{ Total MUFA } & \multicolumn{3}{|c|}{ Total n-6 PUFA } \\
\hline & 1.0 & 2.2 & 0.645 & 28.8 & 27.5 & 0.296 & -5.0 & 6.3 & 0.429 & 0.9 & 1.7 & 0.581 \\
\hline & \multicolumn{3}{|c|}{$\mathrm{R}^{2}=0.111, \mathrm{~F}_{11,283}=3.198 ; p=<0.001$ * } & \multicolumn{3}{|c|}{$\mathrm{R}^{2}=0.045, \mathrm{~F}_{11,282}=1.214 ; p=0.277$} & \multicolumn{3}{|c|}{$\mathrm{R}^{2}=0.061, \mathrm{~F}_{11,285}=1.675 ; p=0.078$} & \multicolumn{3}{|c|}{$\mathrm{R}^{2}=0.108, \mathrm{~F}_{11,284}=3.132 ; p=0.001 *$} \\
\hline \multicolumn{13}{|l|}{ Maternal factors in $\mathrm{T} 3$} \\
\hline Maternal Age & 3.2 & 1.1 & 0.004 * & 27.5 & 35.3 & 0.437 & 1.8 & 2.9 & 0.543 & 2.8 & 0.9 & 0.002 \\
\hline Occupation & -3.4 & 10.8 & 0.753 & 204.9 & 342.5 & 0.550 & 24.4 & 28.3 & 0.388 & -0.2 & 9.1 & 0.981 \\
\hline Educational level & 8.8 & 7.6 & 0.246 & -419.4 & 239.4 & 0.081 & -11.1 & 19.9 & 0.578 & 6.4 & 6.4 & 0.314 \\
\hline Ethnicity & 1.6 & 14.5 & 0.915 & -624.9 & 462.4 & 0.178 & -96.8 & 38.4 & $0.015^{*}$ & 5.2 & 12.2 & 0.674 \\
\hline BMI & -0.2 & 1.1 & 0.855 & 49.3 & 34.9 & 0.160 & 6.0 & 2.9 & $0.036 *$ & -0.3 & 0.9 & 0.776 \\
\hline Gestational weight gain & -0.2 & 1.2 & 0.876 & 11.3 & 39.0 & 0.773 & 3.1 & 3.6 & 0.384 & -0.2 & 1.0 & 0.825 \\
\hline Smoking & 4.3 & 6.5 & 0.506 & -26.4 & 207.1 & 0.899 & 17.7 & 17.2 & 0.303 & 3.0 & 5.5 & 0.582 \\
\hline Alcohol consumption & -86.9 & 84.8 & 0.306 & -1756.6 & 2690.1 & 0.514 & -161.6 & 221.9 & 0.467 & -62.4 & 71.7 & 0.385 \\
\hline Physical Activity & -10.4 & 8.4 & 0.215 & 4.8 & 266.1 & 0.986 & -7.4 & 21.9 & 0.737 & -7.2 & 7.1 & 0.307 \\
\hline \multirow[t]{2}{*}{ Diet Quality } & 3.6 & 1.9 & 0.055 & 38.9 & 60.0 & 0.517 & -7.5 & 5.0 & 0.133 & 3.1 & 1.6 & 0.050 * \\
\hline & \multicolumn{3}{|c|}{$\mathrm{R}^{2}=0.082, \mathrm{~F}_{11,286}=2.331 ; p=0.009 *$} & \multicolumn{3}{|c|}{$\mathrm{R}^{2}=0.037, \mathrm{~F}_{11,283}=0.996 ; p=0.450$} & \multicolumn{3}{|c|}{$\mathrm{R}^{2}=0.065, \mathrm{~F}_{11,283}=1.792 ; p=0.049 *$} & \multicolumn{3}{|c|}{$\mathrm{R}^{2}=0.077, \mathrm{~F}_{11,287}=2.170 ; p=0.016^{*}$} \\
\hline
\end{tabular}

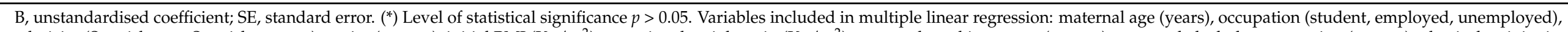

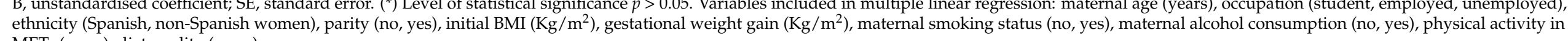
METs (score), diet quality (score). 


\section{Discussion}

The results of present study provide a reference range for the vast majority of serum circulating FAs and FAs ratios in 479 healthy pregnant women from the ECLIPSES study [28], assessed both in $\mathrm{T} 1$ and $\mathrm{T} 3$ of gestation. These results complement the few studies on reference ranges of FAs that exist in pregnant women for different moments of gestation, and add a new measurement with FAs index. Furthermore, this study describes the variation of FA levels in serum according to some maternal factors such as age, obesity, sedentary lifestyle, lower educational level, low diet quality, parity and non-Spanish ethnicity.

In general, any laboratory test is compared with reference ranges in order to assess the degree of compliance with normality levels, both individually in the clinical follow-up of the subject and collectively in epidemiological studies [9]. Adequate levels of FAs in T1 are of great importance considering the part they play in the onset of neurogenesis $[7,8]$. EPA and DHA are particularly critical for supporting the development of the central nervous system, retinal photoreceptors and immune system [6]. Nevertheless, few investigations have reported reference intervals for serum individualized FA concentrations, mainly due to the technical complexity of FAs evaluation compared to other nutrients or biomarkers. In the scarce literature available, FA reference ranges for serum are reported in non-pregnant adults [10-13]. Geographical region, sex, age, physio-pathological conditions [13,22] such as pregnancy [17] and dietary pattern have been seen to lead to some discrepancies. Kish-Trier et al. [40] and Mayo Clinic [12] separately provided reference intervals in age groups from the North American population with somewhat different results. Abdelmagid et al. [11] also reported significant differences between Canadian male and female adults in such FAs as LA or DHA. For pregnant women, reference values have only been described in a Brazilian cohort of 225 healthy pregnant women [16] and in a Norwegian cohort of 247 healthy pregnant women [17]. However, the concentration units (relative terms) and type of sample (erythrocyte membrane) evaluated in these two cohorts cannot be compared with our work methodology and our results. In this regard, reference intervals should be developed using a more uniform and systematic process. In any case, our study provides new information on the European Mediterranean region with socioeconomic, cultural and dietary characteristics different from the previous ones.

Depending on concentration units, the reference intervals of individual FAs in serum can be expressed as either absolute concentrations or percentages of total FA. To the best of our knowledge, no study has reported serum reference ranges in both types of concentration units in healthy pregnant women. To make our results comparable with those of previous studies, reference ranges were reported as relative concentrations because very little literature reports absolute values in pregnant women. Correlations between FA concentrations expressed in absolute and relative values were low-moderate $(r=0.1-0.5)$ with the exception of C12:0, C16:0, Total SFA and C20:5n-3 for which a strong correlation was observed $(r>0.5)$. Identically to our results, other studies have already noted poor correlations between absolute and relative concentrations [40-43] in non-pregnant adults. It is postulated that differences between percentages and concentrations depended on individual FA-characteristics [43]. Moreover, the difference in the correlation could be due to the fact that the percentage of individual FA is calculated based on the whole set of FAs detected, however absolute concentration of individual FA is not dependent upon the relative abundance of other FAs. For this reason, we believe that the measurement expressed in absolute terms would facilitate comparisons among studies.

Our relative results were compared with those reported in other European studies (Table 3). As expected, in the Mediterranean diet pattern $\Sigma$ total MUFA levels appear to be higher in the study from Spain [19] and lower in non-Spanish countries such as the study from the Netherlands [14,38]. Surprisingly, similar levels of $\Sigma$ total MUFA have also been observed in Germany [15] and in UK [39], which are not characterized by following a Mediterranean pattern diet. DHGLA and AA values were higher in the Netherlands than other countries. Normally, the production of inflammatory mediators such as AA is associated with the course of pregnancy. However, abnormally high levels have been 
related to pathological pregnancy complications such as preeclampsia, premature labor, fetal growth, among others (especially in women with risk factors for overweight and obesity) [27]. Some of these complications occur during T3, however Otto et al. [14] measured FA during T1. Furthermore, certain maternal factors such as obesity predispose to suffer a greater number of complications and the study of Vlaardingerbroek. et al. [38] did not report the BMI of their pregnant women population. In addition to the above, DHA concentrations from the Netherlands are higher than those reported in the other studies. Possible explanations for increased DHA in the Netherlands might be the high intake of n-3 supplements or the higher consumption of seafood per capita [17]. Finally, the differences in the measurement time window, sample size and FA assay protocol could explain the difference found with these authors $[14,15,19,38,39]$. Nevertheless, it is important to note that absolute concentrations are more useful and facilitate comparisons between studies and the recommendation for future studies is to report FA levels as absolute units. Further, absolute values consist in an individual measure that assure an economic analysis compared with relative values that require the measurement of all circulating FAs in serum. In this regard, absolute analysis could be included in routine clinical practice of laboratories and not be limited to research studies.

Regarding the evolution of FA levels between $\mathrm{T} 1$ and T3, we conclude in an article from the ECLIPSES study, that serum FA profile changed during the gestational period: $\Sigma$ total SFA, $\Sigma$ total MUFA and $\Sigma$ total n-6 PUFA increased during pregnancy, whereas essential FAs such AA and EPA decrease and DHA remains unchanged [44]. It is well-known that the composition in serum of essential FAs such n-6 PUFA and n-3 PUFA is closely linked to dietary FA consumption $[18,19]$. However, Volk et al. [45] showed that dietary and plasma SFA and MUFA are not related. In addition, some authors reported likewise to our results that FAs intake and the dietary pattern remained similar throughout pregnancy [14,29]. In this sense, diet would not explain the variations observed in serum FA composition and levels, including the lack of correlation between absolute and relative concentrations in our results, which could be related to other factors in combination with physiological changes in plasma volume during pregnancy.

We found that the women with obesity, sedentary lifestyle, lower educational level and those of non-Spanish ethnicity had higher levels of total SFA, total MUFA and AA, and lower levels of total n-3 PUFA and DHA. Factors such as obesity and sedentary lifestyle, could favor the presence of proinflammatory FAs such as AA. Ma et al. showed that overweight, smoking and alcohol drinking can increased the endogenous synthesis of SFA [46]. Other studies showed that variations in FA concentrations could be affected by the genetically conditions, such as genotype expression of fatty acid desaturase [25,47]. Overall, it is important to note that lifestyle, sociodemographic and genetic factors can affect normal FA reference intervals in pregnancy. Therefore, serum FA measure could be considered as an integration of dietary intake and individual biological response [46]. Consequently, absolute percentiles of FAs for $95 \%$ of the population could assist the evaluation of FA status and the identification of women at risk of either under exposure ( $\leq 2.5 \%$ percentile) or over exposure ( $\geq 97.5 \%$ percentile) to an individual FA.

One of the strengths of this study is that it is the first one to define individual SFA, MUFA, and PUFA reference ranges in absolute and relative units in a large sample of healthy women at the beginning and end of pregnancy. The representativeness of the sample size emphasizes the validity of the proposed reference values in the present study. All data were collected by the same trained researchers, and all FA analyses were performed in the same research laboratory. Furthermore, FAs were analyzed by GC-MS which is the more recently clinically validated method for individual FA quantitation [40] and can be applied for fully automated FA profiling in serum samples during dietary studies [35]. Further, our results would facilitate future research into the role of high/low concentrations of individual FAs in obstetric and fetal pathologies. Nevertheless, it does have several limitations. First, FAs were not analyzed in the second trimester of pregnancy and it would have been interesting to monitor the whole gestational period. Second, we were unable to 
assess all FAs in our sample. One of these was ALA, one of the main n-3 PUFAs, which may explain why the n-3 PUFA relative levels in our study were lower than those in other countries. Third, it is difficult to establish a comparison between countries because the FA profile analyzed in total serum/plasma lipids can vary from different populations, included in the same country; further the measurement of FAs in all circulating lipids differ from FA profile performed in circulating triglycerides, phospholipids or esters of cholesterol.

\section{Conclusions}

The present study provides the serum reference interval for FA levels in a large sample of pregnant women from a Mediterranean region. The data are reported in the first and third trimesters of pregnancy, expressed both as absolute values of their serum concentrations, and as relative values in terms of the percentage of total FA. The percentiles of reference ranges proposed will be a useful tool for assessing the degree of adequacy of FAs in pregnant women, in both individual monitoring of pregnancy, and populationbased epidemiological studies. However, every region must provide their own reference ranges in accordance with the characteristics of their population. Further research is needed to identify the personal and environmental factors that contribute to a healthy diet during pregnancy.

Supplementary Materials: The following are available online at https:/ /www.mdpi.com/article/10 $.3390 / \mathrm{nu} 13114037 / \mathrm{s} 1$, Table S1: Pearson correlations between absolute $(\mu \mathrm{mol} / \mathrm{L})$ and relative $(\%$ of total FAs) concentration of fatty acids in serum.

Author Contributions: Conceptualization, V.A.; formal analysis, C.M.-G. and V.A.; data curation, N.S.O., C.M.-G. and V.A.; Investigation, C.M.-G., R.D. and V.A.; writing-original draft preparation, C.M.-G. and V.A.; writing-review and editing, C.M.-G., R.D. and V.A. All authors have read and agreed to the published version of the manuscript.

Funding: This study was funded by a grant (PI12/02777) from the Health Research Fund of the Ministry of Health and Consumption (Madrid, Spain) [Instituto de Salud Carlos III, Fondo de Investigación Sanitaria, Ministerio de Sanidad y Consumo] and co-funded by European Union (ERDF/ESF, "A way to make Europe" /"Investing in your future"). The funding bodies play no part in the design of the study, collection and interpretation of data, or decision to publish.

Institutional Review Board Statement: The study was conducted according to the guidelines of the Declaration of Helsinki, and approved by the Clinical Research Ethics Committee of the Jordi Gol Research Institute in Primary Care [Instituto de Investigación en Atención Primaria; IDIAP] and the Spanish Agency for Medicines and Health Products [Agencia Española del Medicamento y Productos Sanitarios; AEMPS] (AEMPS IJG-FER-2012 on 9 July 2018).

Informed Consent Statement: Informed consent was obtained from all subjects involved in the study.

Data Availability Statement: The data presented in this study are available on request from the corresponding author. The data are not publicly available due to restrictions of privacy.

Acknowledgments: The authors thank the following entities for their contributions in ECLIPSES study: NUTRISAM Research Group, Universitat Rovira i Virgili (URV), Reus, Spain. Sexual and Reproductive Health Care Services (ASSIR) of Tarragona, Spain. Research support Unit-Tarragona and Central Unit-Barcelona of the Institut d'Atenció Primària IDIAP Jordi Gol, Institut Català de la Salut, and Research Centre for Omic Sciences (COS, Eurecat), Universitat Rovira i Virgili, Reus, Spain.

Conflicts of Interest: The authors declare no conflict of interest.

\section{References}

1. Mousa, A.; Naqash, A.; Lim, S. Macronutrient and micronutrient intake during pregnancy: An overview of recent evidence. Nutrients 2019, 11, 443. [CrossRef]

2. Gernand, A.D.; Schulze, K.J.; Stewart, C.P.; West, K.P.; Christian, P. Micronutrient deficiencies in pregnancy worldwide: Health effects and prevention. Nat. Rev. Endocrinol. 2016, 12, 274-289. [CrossRef] [PubMed]

3. Haggarty, P. Effect of placental function on fatty acid requirements during pregnancy. Eur. J. Clin. Nutr. 2004, 58, 1559-1570. [CrossRef] [PubMed] 
4. Hoge, A.; Tabar, V.; Donneau, A.-F.; Dardenne, N.; Degée, S.; Timmermans, M.; Nisolle, M.; Guillaume, M.; Castronovo, V. Imbalance between Omega-6 and Omega-3 Polyunsaturated Fatty Acids in Early Pregnancy Is Predictive of Postpartum Depression in a Belgian Cohort. Nutrients 2019, 11, 876. [CrossRef] [PubMed]

5. Huang, Y.; Iosif, A.-M.; Hansen, R.L.; Schmidt, R. Maternal polyunsaturated fatty acids and risk for autism spectrum disorder in the MARBLES high-risk study. Autism 2020, 24, 1191-1200. [CrossRef] [PubMed]

6. Wu, G.; Imhoff-Kunsch, B.; Girard, A.W. Biological Mechanisms for Nutritional Regulation of Maternal Health and Fetal Development. Paediatr. Périnat. Epidemiol. 2012, 26, 4-26. [CrossRef]

7. Hoge, A.; Bernardy, F.; Donneau, A.-F.; Dardenne, N.; Degée, S.; Timmermans, M.; Nisolle, M.; Guillaume, M.; Castronovo, V. Low omega-3 index values and monounsaturated fatty acid levels in early pregnancy: An analysis of maternal erythrocytes fatty acids. Lipids Health Dis. 2018, 17, 1-11. [CrossRef]

8. Lauritzen, L.; Brambilla, P.; Mazzocchi, A.; Harsløf, L.B.S.; Ciappolino, V.; Agostoni, C. DHA Effects in Brain Development and Function. Nutrients 2016, 8, 6. [CrossRef] [PubMed]

9. Clinical and Laboratory Standards Institute (CLSI). Defining, Establishing, and Verifying Reference Intervals in the Clinical Laboratory, 3rd ed.; Clinical and Laboratory Standards Institute: Wayne, PA, USA, 2010.

10. Sera, R.K.; McBride, J.H.; Higgins, S.A.; Rodgerson, D.O. Evaluation of reference ranges for fatty acids in serum. J. Clin. Lab. Anal. 1994, 8, 81-85. [CrossRef]

11. Abdelmagid, S.A.; Clarke, S.E.; Nielsen, D.E.; Badawi, A.; El-Sohemy, A.; Mutch, D.M.; Ma, D.W. Comprehensive profiling of plasma fatty acid concentrations in young healthy Canadian adults. PloS ONE 2015, 10, e0116195. [CrossRef]

12. Mayo Clinic Laboratories, Fatty Acid Profile, Essential, Serum. Available online: https://www.mayocliniclabs.com/test-catalog/ Clinical+and+Interpretive/82426 (accessed on 9 November 2021).

13. Khaw, K.-T.; Friesen, M.D.; Riboli, E.; Luben, R.; Wareham, N. Plasma Phospholipid Fatty Acid Concentration and Incident Coronary Heart Disease in Men and Women: The EPIC-Norfolk Prospective Study. PLoS Med. 2012, 9, e1001255. [CrossRef] [PubMed]

14. Otto, S.J.; Van Houwelingen, A.C.; Badart-Smook, A.; Hornstra, G. Changes in the maternal essential fatty acid profile during early pregnancy and the relation of the profile to diet. Am. J. Clin. Nutr. 2001, 73, 302-307. [CrossRef]

15. Enke, U.; Jaudszus, A.; Schleussner, E.; Seyfarth, L.; Jahreis, G.; Kuhnt, K. Fatty acid distribution of cord and maternal blood in human pregnancy: Special focus on individual trans fatty acids and conjugated linoleic acids. Lipids Health Dis. 2011, 10, 247. [CrossRef] [PubMed]

16. Pinto, T.J.P.; Farias, D.R.; Rebelo, F.; Lepsch, J.; Vaz, J.S.; Moreira, J.D.; Cunha, G.M.; Kac, G. Lower Inter-Partum Interval and Unhealthy Life-Style Factors Are Inversely Associated with n-3 Essential Fatty Acids Changes during Pregnancy: A Prospective Cohort with Brazilian Women. PLoS ONE 2015, 10, e0121151. [CrossRef]

17. Araujo, P.; Kjellevold, M.; Nerhus, I.; Dahl, L.; Aakre, I.; Moe, V.; Smith, L.; Markhus, M.W. Fatty Acid Reference Intervals in Red Blood Cells among Pregnant Women in Norway-Cross Sectional Data from the 'Little in Norway' Cohort. Nutrients 2020, 12, 2950. [CrossRef]

18. Mazzocchi, A.; Leone, L.; Agostoni, C.; Pali-Schöll, I. The Secrets of the Mediterranean Diet. Does [Only] Olive Oil Matter? Nutrients 2019, 11, 2941. [CrossRef]

19. Montes, R.; Chisaguano, A.M.; I Castellote, A.; Morales, E.; Sunyer, J.; López-Sabater, M.C. Fatty-acid composition of maternal and umbilical cord plasma and early childhood atopic eczema in a Spanish cohort. Eur. J. Clin. Nutr. 2013, 67, 658-663. [CrossRef] [PubMed]

20. Kohlmeier, L. Future of dietary exposure assessment. Am. J. Clin. Nutr. 1995, 61, 702S-709S. [CrossRef]

21. Bradbury, K.E.; Skeaff, C.M.; Crowe, F.L.; Green, T.J.; Hodson, L. Serum Fatty Acid Reference Ranges: Percentiles from a New Zealand National Nutrition Survey. Nutrients 2011, 3, 152-163. [CrossRef] [PubMed]

22. Zhao, J.P.; Levy, E.; Shatenstein, B.; Fraser, W.D.; Julien, P.; Montoudis, A.; Spahis, S.; Xiao, L.; Nuyt, A.M.; Luo, Z.C. Longitudinal circulating concentrations of long-chain polyunsaturated fatty acids in the third trimester of pregnancy in gestational diabetes. Diabet. Med. 2015, 33, 939-946. [CrossRef]

23. Ohnishi, H.; Saito, Y. Eicosapentaenoic Acid (EPA) Reduces Cardiovascular Events: Relationship with the EPA/Arachidonic Acid Ratio. J. Atheroscler. Thromb. 2013, 20, 861-877. [CrossRef] [PubMed]

24. Christian, L.M.; Blair, L.M.; Porter, K.; Lower, M.; Cole, R.M.; Belury, M.A. Polyunsaturated Fatty Acid (PUFA) Status in Pregnant Women: Associations with Sleep Quality, Inflammation, and Length of Gestation. PLOS ONE 2016, 11, e0148752. [CrossRef]

25. Hu, Y.; Tanaka, T.; Zhu, J.; Guan, W.; Wu, J.; Psaty, B.M.; McKnight, B.; King, I.B.; Sun, Q.; Richard, M.; et al. Discovery and fine-mapping of loci associated with MUFAs through trans-ethnic meta-analysis in Chinese and European populations. J. Lipid Res. 2017, 58, 974-981. [CrossRef] [PubMed]

26. Gibson, R.A.; Muhlhausler, B.; Makrides, M. Conversion of linoleic acid and alpha-linolenic acid to long-chain polyunsaturated fatty acids (LCPUFAs), with a focus on pregnancy, lactation and the first 2 years of life. Matern. Child Nutr. 2011, 7, 17-26. [CrossRef]

27. Szczuko, M.; Kikut, J.; Komorniak, N.; Bilicki, J.; Celewicz, Z.; Ziętek, M. The Role of Arachidonic and Linoleic Acid Derivatives in Pathological Pregnancies and the Human Reproduction Process. Int. J. Mol. Sci. 2020, 21, 9628. [CrossRef] 
28. Arija, V.; Fargas, F.; March, G.; Abajo, S.; Basora, J.; Canals, J.; Ribot, B.; Aparicio, E.; Serrat, N.; Hernández-Martínez, C.; et al. Adapting iron dose supplementation in pregnancy for greater effectiveness on mother and child health: Protocol of the ECLIPSES randomized clinical trial. BMC Pregnancy Childbirth 2014, 14, 33. [CrossRef] [PubMed]

29. Jardí, C.; Aparicio, E.; Bedmar, C.; Aranda, N.; Abajo, S.; March, G.; Basora, J.; Arija, V.; the ECLIPSES Study Group Food Consumption during Pregnancy and Post-Partum. ECLIPSES Study. Nutrients 2019, 11, 2447. [CrossRef]

30. Fernández, R.; Escuriet, R.; Costa, D.; Armelles, M.; Cabezas, C. Protocol de seguiment de l'embaràs a catalunya, Dir. Gen. Salut Pública. 2018. Available online: https://salutpublica.gencat.cat/web/.content/minisite/aspcat/promocio_salut/embaras_part_ puerperi/protocol_seguiment_embaras/protocol-seguiment-embaras-2018.pdf. (accessed on 9 November 2021).

31. De Catalunya, G. Classificació Catalana d'Ocupacions (CCO-2011). 2011. Available online: https://www.idescat.cat/serveis/ biblioteca/docs/cat/cco2011.pdf (accessed on 9 November 2021).

32. Edwards, M.K.; Loprinzi, P.D. Affective Responses to Acute Bouts of Aerobic Exercise, Mindfulness Meditation, and Combinations of Exercise and Meditation: A Randomized Controlled Intervention. Psychol. Rep. 2019, 122, 465-484. [CrossRef]

33. World Health Organization (WHO). Obesity and overweight. Available online: https://www.who.int/news-room/fact-sheets/ detail/obesity-and-overweight. (accessed on 9 November 2021).

34. Norte Navarro, A.I.; Ortiz Moncada, R. Calidad de la dieta española según el índice de alimentación saludable. Nutr. Hosp. 2011, 26, 330-336. [CrossRef]

35. Automated Sample Preparation for Profiling Fatty Acids in Blood and Plasma using the Agilent 7693. Available online: https://www.agilent.com/cs/library/applications/5990-4822EN.pdf. (accessed on 9 November 2021).

36. Mowbray, F.I.; Fox-Wasylyshyn, S.M.; El-Masri, M.M. Univariate outliers: A conceptual overview for the nurse researcher. Can. J. Nurs. Res. 2019, 51, 31-37. [CrossRef]

37. Cousineau, D.; Chartier, S. Outliers detection and treatment: A review. Int. J. Psychol. Res. 2010, 3, 58-67. [CrossRef]

38. Vlaardingerbroek, H.; Hornstra, G. Essential fatty acids in erythrocyte phospholipids during pregnancy and at delivery in mothers and their neonates: Comparison with plasma phospholipids. Prostaglandins, Leukot. Essent. Fat. Acids 2004, 71, 363-374. [CrossRef] [PubMed]

39. Wheeler, S.J.; Poston, L.; Thomas, J.E.; Seed, P.T.; Baker, P.N.; Sanders, T.A.B. Maternal plasma fatty acid composition and pregnancy outcome in adolescents. Br. J. Nutr. 2011, 105, 601-610. [CrossRef] [PubMed]

40. Kish-Trier, E.; Schwarz, E.; Pasquali, M.; Yuzyuk, T. Quantitation of total fatty acids in plasma and serum by GC-NCI-MS. Clin. Mass Spectrom. 2016, 2, 11-17. [CrossRef]

41. Lagerstedt, S.A.; Hinrichs, D.R.; Batt, S.M.; Magera, M.J.; Rinaldo, P.; McConnell, J.P. Quantitative Determination of Plasma C8-C26 Total Fatty Acids for the Biochemical Diagnosis of Nutritional and Metabolic Disorders. Mol. Genet. Metab. 2001, 73, 38-45. [CrossRef] [PubMed]

42. Sergeant, S.; Ruczinski, I.; Ivester, P.; Lee, T.C.; Morgan, T.M.; Nicklas, B.J.; Mathias, R.A.; Chilton, F.H. Impact of methods used to express levels of circulating fatty acids on the degree and direction of associations with blood lipids in humans. Br. J. Nutr. 2016, 115, 251-261. [CrossRef] [PubMed]

43. Mocking, R.J.T.; Assies, J.; Lok, A.; Ruhé, H.G.; Koeter, M.W.J.; Visser, I.; Bockting, C.L.H.; Schene, A.H. Statistical Methodological Issues in Handling of Fatty Acid Data: Percentage or Concentration, Imputation and Indices. Lipids 2012, 47, 541-547. [CrossRef]

44. Aparicio, E.; Martín-Grau, C.; Hernández-Martinez, C.; Voltas, N.; Canals, J.; Arija, V. Changes in fatty acid levels (saturated, monounsaturated and polyunsaturated) during pregnancy. BMC Pregnancy Childbirth 2021. in process of being published.

45. Volk, B.M.; Kunces, L.J.; Freidenreich, D.J.; Kupchak, B.R.; Saenz, C.; Artistizabal, J.C.; Fernandez, M.L.; Bruno, R.; Maresh, C.M.; Kraemer, W.J.; et al. Effects of Step-Wise Increases in Dietary Carbohydrate on Circulating Saturated Fatty Acids and Palmitoleic Acid in Adults with Metabolic Syndrome. PLoS ONE 2014, 9, e113605. [CrossRef]

46. Ma, J.; Folsom, A.R.; Shahar, E.; Eckfeldt, J.H. Plasma fatty acid composition as an indicator of habitual dietary fat intake in middle-aged adults. The Atherosclerosis Risk in Communities (ARIC) Study Investigators. Am. J. Clin. Nutr. 1995, 62, 564-571. [CrossRef]

47. Wilson, N.; Mantzioris, E.; Middleton, P.; Muhlhausler, B. Influence of sociodemographic, lifestyle and genetic characteristics on maternal DHA and other polyunsaturated fatty acid status in pregnancy: A systematic review. Prostaglandins Leukot. Essent. Fat. Acids 2020, 152, 102037. [CrossRef] [PubMed] 